\title{
Clinical and Quality-of-Life Outcomes after Autologous Fascial Sling and Tension-Free Vaginal Tape: A Prospective Randomized Trial
}

\author{
Joao L. Amaro, Hamilto Yamamoto, Paulo R. Kawano, Guilherme Barros, Monica O. O. Gameiro, \\ Aparecido D. Agostinho
}

Department of Urology (JLA, HY, PRK, ADA), Department of Anesthesia (GB) and Section of Physiotherapy (MOOG), School of Medicine, UNESP, Botucatu, Sao Paulo, Brazil

\begin{abstract}
Purpose: Evaluate the impact autologous fascial sling (AFS) and tension-free vaginal tape (TVT) procedures on qualityof-life in incontinent women.

Materials and Methods: Forty-one women were randomly distributed into two groups. Group G1 (n=21), underwent AFS and group G2 ( $\mathrm{n}=20)$ TVT implant. The clinical follow up was performed at 1, 6, 12 and 36 months.

Results: TVT operative time was significantly shorter than AFS. Cure rates were 71\% at 1 month, $57 \%$ at 6 and 12 months in G1. In G2, cure rates were $75 \%$ at 1 month, $70 \%$ at 6 months and $65 \%$ at 12 months; there was no significant difference between groups. As regards the satisfaction rate, there was no statistical difference between groups. Analysis of quality of life at 36 months revealed that there was no significant difference between groups.

Conclusion: Similar results between AFS and TVT, except for operative time were shorter in TVT.
\end{abstract}

Key words: urinary incontinence, stress; suburethral slings; tensionless vaginal tape; random allocation Int Braz J Urol. 2009; 35: 60-7

\section{INTRODUCTION}

Urinary incontinence (UI) can have a severe impact on quality of life (QoL). Thus, assessing the effect of interventions is of relevance. QoL is a multidimensional concept reflecting an individual's experience of physical, emotional and social well being, as well as perceptions of health status (1). In general, UI has been shown to affect the psychological occupational, domestic and sexual lives of $15 \%$ to $30 \%$ of women at all ages (2). QoL is an abstract and highly subjective concept influenced by personal and cultural values, beliefs, self concepts, goals, age and life expectancy. It is usually measured using structured questionnaires containing a variable number of fields. There are two major types of QoL questionnaire, generic and disease specific. Unfortunately, the generic forms are often used inappropriately in incontinent women (3) and it is necessary to use a disease or condition-specific quality of life measure, e.g. the King's Health Questionnaire (KHQ) (4).

Sling procedures have been used for treatment of urinary incontinence in women since the beginning of the $20^{\text {th }}$ century (5). Indications for biologic grafts include patients with a history of poor vaginal healing, pelvic bone trauma, pelvic radiation therapy, or urethral reconstruction or are based on surgeon's preference (6). Success rates of autologous graft used 
for mid-urethral slings range from $70-91 \%$ at $33-52$ months (7).

Tension-free vaginal tape (TVT) has been introduced as a minimal invasive sling procedure for treatment of stress urinary incontinence (SUI) with a high success rate (8).

The aim of this study was to evaluate the impact of autologous fascial sling (AFS) and TVT procedures on QoL in incontinent women.

\section{MATERIALS AND METHODS}

Between January 2001 and March 2002, 41 women, from the Urogynecology Outpatient Clinic of Botucatu Medical School - UNESP, with a principal complaint of stress urinary incontinence were studied in a prospective trial. These patients were randomly distributed into two groups. Group G1 $(n=21)$ was submitted to AFS and Group G2 $(\mathrm{n}=20)$ to TVT implant. The randomization followed a blind raffle where the procedures (TVT and Sling) were written on small pieces of paper, folded and placed into a closed box. It was opened just before the surgery when the medical team found out which procedure would be performed. Mean age in G1 was 49 years (range 2669 ), and in G2, 52 years (range 26-79). All surgical procedures were performed by the same surgeon. This study was approved by the Bioethics Committee of Botucatu Medical School - UNESP.

Before surgery, stress urinary incontinence was urodynamically confirmed (Dynograph R.611 recorder) in all study participants. The following parameters were investigated: free maximum urinary flow $\left(\mathrm{Q}_{\max }\right)$, maximum detrusor pressure $\left(\mathrm{P}_{\text {det.max }}\right)$ and maximum urinary flow $\left(\mathrm{Q}_{\max }\right)$ during voiding. Valsalva leak-point pressure (VLPP) was obtained when the patient, in lithotomy position, reported the desire to void due to maximum bladder capacity. Detrusor overactivity was defined as the presence of spontaneous or provoked involuntary detrusor contractions during vesical filling phase, whether partially suppressed or not (9). Bladder outlet obstruction was considered when $\mathrm{P}_{\text {det }} / \mathrm{Q}_{\max }$ exceeded $20 \mathrm{~cm} \mathrm{H}_{2} \mathrm{O}$ with maximum flow rate $\left(\mathrm{Q}_{\text {max }}\right)$ below 12 $\mathrm{mL}$ per second (10). Patients with involuntary detrusor contractions or preexisting bladder outlet obstruction during urodynamic analysis were excluded.
The clinical follow-up was performed and subjective success rate was evaluated in a transversal cut off at 1, 6, and 12 months and then annually after hospital discharge. A questionnaire was used to obtain personal data, obstetric, gynecologic, family medical history, and subjective analysis of urine loss. Cure was defined as complete dryness with no usage of pads as reported by the patient.

De novo urgency was defined as the postoperative development of symptoms of urgency, which were not present before surgery and persisted for more than 1 month. These symptoms were based on clinical evaluation.

Cure rate, long-term patients' satisfaction and impact on QoL were performed at 36 months after surgery. For QoL evaluation, a validated questionnaire (11) (King's Health Questionnaire) and personal interviews were conducted. The questionnaire consists of 4 sections, the first contains 2 domains that measure female perception of general health and the impact of urinary symptoms on life. The second section contains 14 questions that allow women to rate the impact of urinary symptoms on 5 other QoL fields, namely role limitations, physical/social limitations, personal relationships, emotions and sleep and energy. The third section assesses the severity of measures associated with urinary incontinence. The fourth section consists of a separate scale for rating different urinary symptoms, including stress incontinence. Scores in each domain range between zero and 100, a higher score indicating a greater impairment of QoL (4). Follow-up ranged from 36 to 54 months (median: 44 months) as surgeries were performed at the different dates.

Body mass index (BMI) was calculated and classified according to Garrow (12).

All patients underwent physical examination including stress test. The degree of pelvic organ prolapse was assessed and graded according to Baden et al. (13).

Basal laboratory investigations (serum creatinine, complete blood count, chemical and microscopic urinalysis, urine culture) were all routinely performed. In exceptional cases (history of lithiasis, urinary infection) renal ultrasound and plain X-ray of the kidney, ureters and bladder were carried out. 
In the immediate postoperative, intravenous tramadol $\left(10 \mathrm{mg} / \mathrm{mL}^{-1}\right)$ was used in a patient-controlled analgesia (PCA) pump. After an i.v. loading dose of $0.07 \mathrm{~mL} / \mathrm{Kg}^{-1}$ (administered over a period of $30 \mathrm{~min}$ ), a continuous background i.v. infusion was set at $1.5 \mathrm{~mL} / \mathrm{h}^{-1}$ and a demand bolus injection was set at $0.2 \mathrm{~mL}$ (lock out interval of $30 \mathrm{~min}$.). Data of PCA demand, dose delivered and total analgesic consumption were retrieved from the PCA computer data bank.

\section{Operative Technique}

Autologous fascial sling was carried-out as previously described with some modifications (14). A transverse suprapubic incision for withdrawal of the rectus fascia strip $(10 \times 2 \mathrm{~cm})$ and aponeurosis closure was done with 1-vicryl thread. The strip was prepared and both its edges were tied with 0 -prolene, which was left long. A Foley catheter was used to empty the bladder. A submucosal saline injection was performed on the anterior vaginal wall and a longitudinal incision was performed $2 \mathrm{~cm}$ from the urethral orifice. Dissection of the vaginal mucosa was done until identification of the retropubic space. Then the strip of rectus fascia was positioned with the aid of Stamey needle around the middle urethra maintaining the strip without tension. The wires were approximated in the midline and the sling was fixed to the underlying peri-urethral fascia using 4-0 catgut sutures at the 6 and 12 o'clock positions. The vaginal mucosa and the suprapubic skin incision were then closed. Cystoscopy was performed in all patients submitted to autologous sling.

The TVT procedure was performed as described by Ulmsten et al., except that the operation was carried out under spinal anesthesia (8). Cystoscopy was performed in all patients.

Foley catheter was left indwelling for 1 day in all patients after anti-incontinence surgery. The following parameters were postoperatively evaluated: operativeroom time, objective postoperative pain, complications, length of hospital stay, postoperative catheterization, and time to return to normal activities.

The analysis of clinical and urodynamic characteristics were performed using the Mann-Whitney non-parametric test for quantitative variables (15) and the Godman test as regards the categorical ones (16).
Differences were considered significant for $\mathrm{p}$ value $<$ 0.05 .

\section{RESULTS}

No statistically significant difference in the demographic data and urodynamic parameters were preoperatively observed between groups (Table-1). Operative time was significantly shorter than TVT than with AFS. There was no statistical difference between groups in bladder injuries, hospitalization time, post-operative catheterization and return to normal activities (Table-2). There was neither prolonged urine retention nor other complications in both groups.

Cure rates were $71 \%$ at 1 month, $57 \%$ at 6 and 12 months, and $55 \%$ at 36 months in $\mathrm{G} 1$, whereas in $\mathrm{G} 2$ they were $75 \%$ at 1 month, $70 \%$ at 6 months, $65 \%$ at 12 months and $63 \%$ at 36 months, with no significant difference between groups.

Although 2 patients, one from each group, died from other diseases within the 36-month following period, the post-operative satisfaction rate was $80 \%$ in $\mathrm{G} 1$ and $58 \%$ in $\mathrm{G} 2$, with no statistical difference between groups (Table-3). However, in AFS group, the satisfaction rate was 62.5 to $97.5 \%$, while in TVT group it was between 36 to $80 \%$. A 95\% confidence interval was used for satisfaction assessment.

De novo urgency symptoms were observed in $40 \%$ of the patients in G1 and in $42 \%$ of those in G2 at 36 months with no difference between groups.

Condition-specific QoL postoperatively assessed by the King's Health Questionnaire (KHQ) did not significantly differ between groups (Table-4).

The comparison between satisfaction rate and condition-specific QoL contained in the KHQ showed a significant correlation between these variables demonstrating that unsatisfied patients had higher scores in all different fields, except in perception of general health.

\section{COMMENTS}

In our study, the initial demographic and urodynamic data demonstrated homogeneity between the groups. 
Table 1 - Preoperative clinical and urodynamics characteristics of the patients submitted to autologous fascial sling (AFS) $(n=21)$ and tension-free vaginal tape $(T V T)(n=20)$.

\begin{tabular}{lccc}
\hline Variables & \multicolumn{2}{c}{ Group } & Statistics \\
\hline Mean age (range) & $49(26-69)$ & $52(26-79)$ & $0.38(\mathrm{p}>0.05)$ \\
Mean of body mass index $\left(\mathrm{k} / \mathrm{m}^{2}\right)($ range $)$ & $30.2(22-34)$ & $28.2(24-42)$ & $1.37(\mathrm{p}>0.05)$ \\
Mean of parity (range) & $4(1-9)$ & $4(1-12)$ & $0.48(\mathrm{p}>0.05)$ \\
Mean vaginal deliveries (range) & $4(1-8)$ & $4(0-11)$ & $0.12(\mathrm{p}>0.05)$ \\
Previous incontinence surgery & $4(19 \%)$ & $6(30 \%)$ & $0.82(\mathrm{p}>0.05)$ \\
Hysterectomy & $1(4.7 \%)$ & $4(20 \%)$ & $1.51(\mathrm{p}>0.05)$ \\
Abdominal leak point pressure $\left(\mathrm{cm} \mathrm{H}_{2} \mathrm{O}\right)($ range $)$ & $99(50-154)$ & $95.5(64-157)$ & $0.34(\mathrm{p}>0.05)$ \\
Mean free maximum urinary flow $(\mathrm{mL} / \mathrm{s})($ range) & $30(17-57)$ & $33(13-65)$ & $0.86(\mathrm{p}>0.05)$ \\
Mean of maximum detrusor pressure $\left(\mathrm{cm} \mathrm{H}_{2} \mathrm{O}\right)($ range $)$ & $29(14-48)$ & $25(13-37)$ & $1.08(\mathrm{p}>0.05)$ \\
Mean of maximum volume (mL) (range) & $398(285-700)$ & $406(200-678)$ & $0.21(\mathrm{p}>0.05)$ \\
\hline
\end{tabular}

In the immediate postoperative period, there were no differences between the groups, except for operating time, which was significantly shorter in the TVT group. Similar outcomes were observed in relation to this parameter; however, no additional risk associated with this finding was observed (17).

Table 2 - Outcome of autologous fascial sling (AFS) and tension-free vaginal tape (TVT).

\begin{tabular}{lccc}
\hline Variables & AFS & TVT & Statistics \\
\hline Mean operative time (min) (range) & $70(45-105)$ & $33(25-70)$ & $4.80(\mathrm{p}<0.05)$ \\
Mean dosage of analgesics (mg) (range) & $142(50-473)$ & $85(15-269)$ & $1.78(\mathrm{p}>0.05)$ \\
Bladder injuries & $1(4.8 \%)$ & $2(10 \%)$ & $0.64(\mathrm{p}>0.05)$ \\
Mean of hospitalization time (h) (range) & $24(24-48)$ & $24(24-48)$ & $0.63(\mathrm{p}>0.05)$ \\
Mean of postoperative catheterization (h) (range) & $24(12-48)$ & $24(12-72)$ & $0.16(\mathrm{p}>0.05)$ \\
Mean days to return to normal activities (range) & $30(3-90)$ & $30(4-90)$ & $0.16(\mathrm{p}>0.05)$ \\
\hline
\end{tabular}


Autologous Fascial Sling and Tension-Free Vaginal Tape

Table 3 - Satisfaction rate of autologous fascial sling (AFS) and tension-free vaginal tape (TVT) at 36-month follow up.

\begin{tabular}{lccc}
\hline \multirow{2}{*}{ Group } & \multicolumn{2}{c}{ Satisfaction Rate } & Statistics \\
\hline AFS & Unsatisfied (\%) & Satisfied (\%) & $2.24(\mathrm{p}>0.05)$ \\
TVT & 20 & 80 & 58 \\
\hline
\end{tabular}

Song et al. (18) observed a shorter operating time and quicker recovery in TVT group. Despite this, in the postoperative analgesia using PCA pump did not statistically differ between groups in this study, showing that there was no additional discomfort associated to the techniques used.

In general, cure rate may be based on a great variety of parameters, some of them seem to be very lenient whereas others are relatively subjective. Therefore, it may be more appropriate to report each selected variable separately in order to accurately convey true outcomes. In our series, cure rate was considered as complete dryness with no usage of pads, and similar results were observed in both groups. Applying the same cure criteria in a prospective randomized study, Wadie at al. (17) observed short-term success rates with AFS and TVT
$(92 \%)$ which were higher than those encountered in this study.

Urgency incontinence is usually more bothersome for women than stress urinary incontinence. Women with de novo urgency are significantly older, have higher BMI, and higher parity than those without it (19). Damage to bladder autonomic denervation has been suggested as one of the causes of de novo urgency, which could be due to extensive bladder dissection for pubovaginal sling in contrast with TVT procedure that required little dissection (20). However, incidence of de novo urgency was similar both groups studied here. Nonetheless, given that de novo urgency is more bothersome, this fact might have similarly interfered with the quality of life of patients in both groups. Some authors observed $4 \%$ of de novo detrusor overactivity with AFS and no cases

Table 4 - Postoperative evaluation of condition-specific quality of life King's Health Questionnaire (KHQ) of the patients submitted to autologous fascial sling (AFS) and tension-free vaginal tape (TVT) at 36-month evaluation.

\begin{tabular}{|c|c|c|c|}
\hline Domain of KHQ & AFS & TVT & Statistics \\
\hline Median of score general health (range) & $50.00(0-100)$ & $50.00(0-75)$ & $0.39(\mathrm{p}>0.05)$ \\
\hline Median of score incontinence Impact (range) & $33.34(0-100)$ & $0.00(0-100)$ & $0.19(p>0.05)$ \\
\hline Median of score role limitations (range) & $0.00(0-83.33)$ & $0.00(0-100)$ & $0.93(p>0.05)$ \\
\hline Median of score physical limitations (range) & $0.00(0-100)$ & $0.00(0-100)$ & $0.96(p>0.05)$ \\
\hline Median of score social limitations (range) & $5.56(0-100)$ & $0.00(0-66.67)$ & $0.81(\mathrm{p}>0.05)$ \\
\hline Median of score personal relationships (range) & $0.00(0-100)$ & $0.00(0-100)$ & $0.69(\mathrm{p}>0.05)$ \\
\hline Median of score emotions (range) & $0.00(0-100)$ & $0.00(0-100)$ & $0.87(\mathrm{p}>0.05)$ \\
\hline Median of score sleep (range) & $25.00(0-100)$ & $0.00(0-100)$ & $0.34(\mathrm{p}>0.05)$ \\
\hline Median of score severity perception of UI (range) & $16.67(0-86.67)$ & $26.57(0-100)$ & $0.94(\mathrm{p}>0.05)$ \\
\hline
\end{tabular}


with TVT implant (20). It is noteworthy that in this study the diagnosis of de novo urgency was based on clinical rather than urodynamic criteria, and this might explain the higher incidence observed.

Long-term satisfaction rate did not statistically differ between groups. Despite the higher satisfaction rate observed in the AFS group ( $80 \%$ versus $58 \%, \mathrm{p}>0.05$ ), even if more samples were included in this study, the results would likely show significantly higher satisfaction rate in AFS group.

In long-term evaluation of QoL no statistical difference was observed between groups. This study assessed the effect of AFS versus TVT on QoL in the surgical treatment of UI in women. The measurement of QoL is particularly important in the field of urinary incontinence, given that it is largely a symptom-defined condition. The disease-specific QoL (KHQ) a version validated in Portuguese, classified as recommended, was used (11). The present findings showed that AFS and TVT produced similar improvements in QoL at 36-month study. Morgan et al. (21), using a mailed questionnaire validated to QoL (HRQoL), also observed no significant differences in patients who had undergone AFS or TVT.

The analysis between satisfaction rate and QoL evaluation showed that unsatisfied women presented a higher score in the different fields, except in general health; this demonstrated the importance of using disease-specific QoL when evaluating outcomes in anti incontinence procedures.

\section{CONCLUSION}

In this study, AFS and TVT yielded similar results, except for operating time which was shorter in TVT. This finding is very relevant for developing countries where synthetic slings are often costly. However, further control studies are warranted considering AFS is associated with lower cost and rate of disease transmissions, as well as no rejections when compared with synthetic techniques.

\section{CONFLICT OF INTEREST}

None declared.

\section{REFERENCES}

1. Lose G, Fantl JA, Victor A, Walter S, Wells TL, Wyman J, et al.: Outcome measures for research in adult women with symptoms of lower urinary tract dysfunction. Neurourol Urodyn. 1998; 17: 255-62.

2. Diokno AC, Brock BM, Brown MB, Herzog AR: Prevalence of urinary incontinence and other urological symptoms in the noninstitutionalized elderly. J Urol. 1986; 136: 1022-5.

3. Gill TM, Feinstein AR: A critical appraisal of the quality of quality-of-life measurements. JAMA. 1994; 272: 619-26.

4. Kelleher CJ, Cardozo LD, Khullar V, Salvatore S: A new questionnaire to assess the quality of life of urinary incontinent women. Br J Obstet Gynaecol. 1997; 104: 1374-9.

5. Bidmead J, Cardozo L: Sling techniques in the treatment of genuine stress incontinence. BJOG 2000; 107: 147-56.

6. Amrute KV, Badlani GH: Female incontinence: a review of biomaterials and minimally invasive techniques. Curr Opin Urol. 2006; 16: 54-9.

7. McBride AW, Ellerkmann RM, Bent AE, Melick CF: Comparison of long-term outcomes of autologous fascia lata slings with Suspend Tutoplast fascia lata allograft slings for stress incontinence. Am J Obstet Gynecol. 2005; 192: 1677-81.

8. Ulmsten U, Johnson P, Rezapour M: A three-year follow up of tension free vaginal tape for surgical treatment of female stress urinary incontinence. Br J Obstet Gynaecol. 1999; 106: 345-50.

9. Elbadawi A, Yalla SV, Resnick NM: Structural basis of geriatric voiding dysfunction. III. Detrusor overactivity. J Urol. 1993; 150: 1668-80.

10. Axelrod SL, Blaivas JG: Bladder neck obstruction in women. J Urol. 1987; 137: 497-9.

11. Tamanini JT, D'Ancona CA, Botega NJ, Rodrigues Netto N Jr: Validation of the Portuguese version of the King's Health Questionnaire for urinary incontinent women. Rev Saude Publica. 2003; 37: 203-11.

12. Garrow JS.Treatment of obesity: Lancet. 1992; 340: 409-13.

13. Baden WF, Walker TA, Lindsey JH: The vaginal profile. Tex Med. 1968; 64: 56-8.

14. Blaivas JG, Jacobs BZ: Pubovaginal fascial sling for the treatment of complicated stress urinary incontinence. J Urol. 1991; 145: 1214-8.

15. Zar JH: Biostatistical analysis. New Jersey, PrenticeHall. Prentice-Hall. 1999; vol.1; p.122-60. 
16. Goodman LA: Simultaneous confidence intervals for contrast among multinomial populations. Annals of Mathematical Statistics. 1964; 35: 716-25.

17. Wadie BS, Edwan A, Nabeeh AM: Autologous fascial sling vs polypropylene tape at short-term followup: a prospective randomized study. J Urol. 2005; 174: 990-3.

18. Song YF, Huang HJ, Xu B, Hao L: Comparative study of tension-free vaginal tape and fascia lata for stress urinary incontinence. Zhonghua Fu Chan Ke Za Zhi. 2004; 39: 658-61.

19. Holmgren C, Nilsson S, Lanner L, Hellberg D: Frequency of de novo urgency in 463 women who had undergone the tension-free vaginal tape (TVT)

\section{Correspondence address:}

Dr. João Luiz Amaro

Departamento de Urologia

Faculdade de Medicina de Botucatu

Botucatu, SP, 18618-970, Brazil

Fax: + 5514 3811-6271

E-mail: jamaro@fmb.unesp.br procedure for genuine stress urinary incontinence--a long-term follow-up. Eur J Obstet Gynecol Reprod Biol. 2007; 132: 121-5.

20. Kershen RT, Appell RA: De novo urge syndrome and detrusor instability after anti-incontinence surgery: current concepts, evaluation, and treatment. Curr Urol Rep. 2002; 3: 345-53.

21. Morgan DM, Dunn RL, Fenner DE, Faerber G, DeLancey JO, McGuire EJ, et al.: Comparative analysis of urinary incontinence severity after autologous fascia pubovaginal sling, pubovaginal sling and tension-free vaginal tape. J Urol. 2007; 177: 604-8; discussion 608-9.
Accepted after revision: September 9, 2008

\section{EDITORIAL COMMENT}

The authors present a randomized study, with mid-term follow-up, comparing autologous versus synthetic retropubic sling to treat patients with urodynamic stress urinary incontinence. It is a very important study, since it compares the use of an autologous fascia, which has some morbidity related to the harvesting process, and a synthetic material, which may have the inconvenience of being a foreign body. The main questions regarding these two approaches are related to cure rate, morbidity, complications and costs. There is a lack of well-designed randomized series to clearly answer those questions. The use of synthetic material has great acceptance for the physicians, since it decreases the surgery time, avoids the harvesting process and seems to have similar cure rates as fascial slings. On the other hand, the fascial slings have passed the test of time and may have lower cost. The procedure cost is a very important subject. In the present article, the authors have demonstrated that both procedures have similar outcome, with cure rates at 36 months of $55 \%$ on fascial sling group and $63 \%$ on TVT group. However, the surgical time was double in the sling procedure. Thus, other similar studies should be designed to compare the final cost for extra time in the operative room and the cost of using synthetic sling.

In the present study, the authors performed a good quality randomization that is illustrated by the similarity on demographics. However, the urinary incontinence impact on quality of life (QoL) was not evaluated pre-operatively. Since we do not have this information, it creates a bias in the results. Thus, we 
do not know if patients had similar scores at baseline and we do not know how much they improve after surgery regarding their QoL.

The authors determined that pain was similar in both groups. Usually, patients who undergo fascia harvesting have significant pain in the surgical site. The authors evaluate the postoperative pain by the dosage of analgesics delivered, which was almost double in the fascial sling group. The sample size may be a reason why the authors did not find any statistical difference in the analgesic use. Furthermore, the fact that patient did not ask for medication did not exclude the fact that they were experiencing pain. Thus, to draw any conclusion regard pain, it would be necessary to additionally apply a pain visual analogical scale and include more patients in the analysis.

It is interesting to note that $40 \%$ of patients who underwent TVT procedure were unsatisfied, while on the fascial sling group only $20 \%$ were unsatisfied.
This is very concerning. The authors more likely did not find a significant statistical difference between groups because of the small sample size $(n=20)$. The difference may be associated with those patients that were not "dry" but had significant improvement (> $50 \%$ ) on the symptoms, since most of those patients with significant improvement did not need or want alternative treatment and, usually, had an improvement in QOL. Unfortunately, it this not described in the article. Interestingly, when we examine the King's Health Questionnaire to evaluate the quality of life at 36 months post operatively, the median is zero in the majority of fields, which suggests that the majority of the patients are completely satisfied.

There are several questions to be addressed regarding surgical stress urinary incontinence treatment. Similar randomized studies should be done to clearly determine which procedure has the best costefficiency with greater safety and lower morbidity.

Dr. Fernando G. Almeida Section of Urology Federal University of Sao Paulo Sao Paulo, SP, Brazil E-mail:fernandourologia@hotmail.com 